\title{
A Proposal of Early Diagnosis and Early Management in Dengue Infection and Possible COVID-19
}

\author{
Manote Arpornsuwan ${ }^{*}{ }^{*}$ and Matinun Arpornsuwan ${ }^{2}$ \\ ${ }^{1}$ Clinic Dr. Manote, Buriram 31000, Thailand; ${ }^{2}$ Nonthaburi 11120, Thailand
}

\begin{abstract}
Dengue infection may lead to various sequels, such as undifferentiated fever, dengue fever, or dengue hemorrhagic fever, progressive illness, and death. It also causes significant economic burden associated with healthcare costs and loss of labor. Usually, the treatment of dengue infection is supportive therapy and not a specific treatment, while it is often clinically difficult to predict whether dengue-infected patients will progress to severe disease or not. We propose integrated management strategies for early dengue virus infection based on our 5-year experience in early management of dengue infection, literature reviews, and research studies (over the last 10 years). This proposal consists of 4 aspects: (1) rehydration with oral rehydration therapy; (2) reduction of the proinflammatory cytokines, including tumor necrosis factor- $\alpha$ and interleukin- 6 with oral cytokine inhibitors (i.e. pentoxifylline and doxycycline); (3) inhibition of dengue virus replication, viral load reduction, nonstructural protein 1 antigen clearance with doxycycline and ivermectin; and (4) restoration of the immune function by vitamin $D$ and zinc supplementation. The major benefit of these drugs and supplements is that they are already approved by regulatory bodies, affordable, and clinically safe. They result in better clinical and laboratory outcomes, including reductions in hospitalization and cost of treatment. Another benefit is that this strategy may be used for other virus-induced hypercytokinemia, such as coronavirus disease and Ebola.
\end{abstract}

Introduction

Currently, dengue virus (DENV) is the most prevalent of all the arboviruses in the world, with more than 128 countries reporting dengue outbreaks, and nearly 4 billion people living in at-risk

Keywords: Dengue; COVID-19; Early diagnosis; Early management; Pentoxifylline; Doxycycline; Ivermectin; Vitamin D; Zinc.

Abbreviations: ADE, antibody-dependent enhancement; $\mathrm{CBC}$, complete blood count; CD, cluster of differentiation; COVID-19, coronavirus disease; DENV, dengue virus; DF, dengue fever; DHF, dengue hemorrhagic fever; DSS, dengue shock syndrome; EDS, expanded dengue syndrome; ICU, intensive care unit; IL, interleukin; IFN- $\alpha$, interferon-alpha; IFN- $\gamma$, interferon-gamma; IgM, immunoglobulin $\mathrm{M} ; \operatorname{IgG}$, immunoglobulin G; I\$, international dollars; MDMs, monocyte-derived macrophages; mRNA, messenger ribonucleic acid; MERS-CoV, Middle Eastern respiratory syndrome coronavirus; NF- $\mathrm{KB}$, nuclear factor-kappa B; NS1, nonstructural protein 1 ; NS1Ag, nonstructural protein 1 antigen; ORS, oral rehydration solution; ORT, oral rehydration therapy; RDT, rapid diagnostic test; SARS-CoV-2, severe respiratory syndrome coronavirus- 2 ; Th1, Thelper type 1 ; TGF- 13 , transforming growth factor- 1 beta; TNF, tumor necrosis factor; TNF- $\alpha$, tumor necrosis factor-alpha; UF, undifferentiated fever; WHO, World Health Organization.

Received: September 27, 2020; Revised: October 19, 2020; Accepted: November 10, 2020

*Correspondence to: Manote Arpornsuwan, Clinic Dr. Manote, 182/2-3 Soontornthep Rd., Muang Buriram, Buriram 31000, Thailand. ORCID: http://orcid.org/00000001-9689-4157. Tel: +66-44-612262, E-mail: manote_arpornsuwan@yahoo.com

How to cite this article: Arpornsuwan M, Arpornsuwan M. A Proposal of Early Diagnosis and Early Management in Dengue Infection and Possible COVID-19. Exploratory Research and Hypothesis in Medicine 2020;5(4):141-151. doi: 10.14218/ ERHM.2020.00059. areas for dengue infection. ${ }^{1}$ An estimated 390 million people are impacted each year by DENV infection, with 96 million shown to be dengue infection. ${ }^{2}$ Clinical manifestations of dengue infection vary from mild undifferentiated fever (UF) to dengue fever (DF), dengue hemorrhagic fever (DHF), and dengue shock syndrome (DSS), following the World Health Organization (WHO) 1997 dengue guideline. ${ }^{3}$ From the study of children aged 3-14 years-old in Ratchaburi, Thailand, dengue infections were found to cause $12.1 \%$ of febrile illnesses requiring hospitalization, including UF (49.8\%), DF (39.3\%), and DHF (10.9\%) respectively. ${ }^{4}$ The presence of plasma leakage is an important feature in separating DHF from DF. There is no specific treatment for dengue infection, and management is merely supportive care for patients with fluid management during the critical phase, along with ongoing monitoring in the case of DHF. ${ }^{5}$ Mortality is often caused by delays in plasma leakage detection and treatment. Frequently, it is impossible to predict who will have an uneventful defervescence and those who will develop severe dengue. However, the use of WHO warning signs with special care in times of the critical phase can help to identify which patients need more intensive supportive treatment. ${ }^{6}$

Several theories have been proposed for the pathogenesis of DENV infection. DENV infections cause severe abnormal immune stimulation. An abnormal immune response, such as inverted ratio $\mathrm{CD} 4 / \mathrm{CD} 8$, not only reduces the ability of the immune system to eliminate viruses but it also induces excessive cytokine production, which may influence monocytes, endothelial cells, and hepatocytes. Various studies have characterized the monocytes 
as natural DENV host cells and shown these monocytes to be involved in both the pathogenesis and prevention of dengue, such as interferon-alpha (IFN- $\alpha$ ) generation in response to DENV. ${ }^{3-5}$ Also, monocytes/macrophages may produce cytokines and chemokines that reduce the integrity of the endothelial cell layer, ${ }^{7-9}$ which may contribute to plasma leakage, an indication of severe dengue or DHF. ${ }^{10,11}$

In general, high levels of proinflammatory cytokines were measured in patients with severe dengue, including of interleukin (IL)- $1 \beta$, tumor necrosis factor-alpha (TNF- $\alpha$ ), IL-6, IL-8, IL-10, and transforming growth factor- 1 beta (TGF-1ß). The increased levels of TNF- $\alpha$ were associated with greater clinical severity of dengue infection and cases of such may develop disease progression due to excessive inflammation and vascular alterations in patients. ${ }^{12}$ TNF- $\alpha$ is also responsible for increased vascular permeability and hemorrhages by inducing the reactive oxygen and nitrogen intermediates. Plasma increase in TNF- $\alpha$ was associated with decreased blood platelet levels in clinical dengue infection. ${ }^{13}$ The IL-6 level was significantly elevated with severe DENV infection, resulting in fatal outcome in DHF patients as compared to DF patients; therefore, the up-regulation of IL-6 has a significant role towards DHF, suggesting it as a prognostic marker for severe DENV infection. ${ }^{14}$ Another hypothesis for dengue pathogenesis is the antibody-dependent enhancement (ADE), which could explain the finding that serious symptoms of severe dengue occur in children with a secondary DENV infection of a different serotype from the previous one. Previously available antibodies for DENV that cannot be neutralized were the causative factors of the enhancing infection in vitro.

Several studies in vivo and in vitro have found that the tissuedamaging DENV nonstructural protein 1 (NS1) is mainly responsible for the pathophysiological characteristics of severe dengue. ${ }^{9}$ For DHF patients, plasma levels of soluble nonstructural protein 1 antigen $(\mathrm{NS} 1 \mathrm{Ag}$ ) virus products were also higher than in DF patients within the first 72 hours following the onset of fever. ${ }^{15}$ The studies using measurements of plasma viral load have shown that patients with DHF have a greater viral load than patients with DF. ${ }^{10,11}$ During the course of the disease, the maximum viremia tends to occur early.

In the febrile phase, dengue-infected patients develop with highgrade fever immediately and usually take $2-7$ days. During this stage, it is still not clinically known whether the patients diagnosed with dengue infection may progress to severe dengue. Non-severe dengue cases have clinical improvements after defervescence. Some patients have developed progression to the critical stage of plasma leakage without defervescence. However, ultrasonographic evidence of plasma leakage was detected as early as the third day of the illness in some patients, similar to previous studies in children in the DHF case beginning from 2 days before. ${ }^{16}$

\section{Hypothesis}

\section{Early diagnosis and early management in dengue infection with- in the first 24-48 hours after fever onset (before occurrence of plasma leakage) could prevent the development of severe dengue (DHF/DSS) and severe complications}

Generally, dengue fever is a self-limiting illness, with a mortality rate of less than $1 \%$ when diagnosed early and with adequate management. The mortality rate is $2-5 \%$ when severe dengue is diagnosed, but without treatment, the mortality rate can be as high as
$20 \% .{ }^{17}$ Increased levels of cytokines cause the severity of DENV infection; therefore, the reduction of DENV-induced cytokines may be useful for treatment. The problem in practice is whether DHF/DSS may be preventable by inhibiting pro-inflammatory cytokines once diagnosed with DENV infection. There is presently no treatment to improve the clinical symptoms of dengue disease.

We propose, here, early detection, early diagnosis, and management in dengue infection within the first 24-48 hours after fever onset but before the occurrence of plasma leakage, which will prevent the development of severe dengue (DHF/DSS) and severe complications as well as an expanded dengue syndrome (EDS), unusual manifestations of dengue infection with high mortality. Integrated management strategies for early dengue infection consist of 4-dimensional aspects as follows:

1. Rehydration with oral rehydration therapy (ORT) in patients with mild to moderate dehydration from high fever as well as from vomiting.

2. Reduction of the pro-inflammatory cytokines, including TNF- $\alpha$ and IL-6, with oral cytokine inhibitors (i.e. pentoxifylline and doxycycline).

3. Inhibition of DENV replication, viral load reduction, and NS1Ag clearance with doxycycline and ivermectin.

4. Restoring the immune function with vitamin D and zinc supplementation.

A summary of the integrated management strategies for early DENV infection, including the recommended dosages, benefits, and side effects, is shown in Table $1 .^{18}$

Pentoxifylline can reduce the proinflammatory actions of TNF- $\alpha$, and one study has shown the possible use of pentoxifylline in severe dengue. ${ }^{19}$ Treatment with tetracycline or doxycycline has significantly reduced cytokine levels in DHF/DSS patients. ${ }^{20}$ The 3-day treatment with ivermectin showed a considerable difference in clearance time for NS1, even though there was no clinical effectiveness of ivermectin, as seen in that study. ${ }^{21}$ Vitamin D has been demonstrated to regulate immune response and to relieve dengue disease. Vitamin D can activate a down-regulation of proinflammatory cytokine expression, while enhancing the anti-inflammatory cytokine expression. Zinc has many antiviral effects that are recognized by promoting the normal functioning of the innate immune system upon generating both innate and humoral immune responses.

\section{Evaluation of the hypothesis}

\section{Importance of early diagnosis of dengue infection within 24-48 hours after fever onset}

Early and correct diagnosis of dengue infection is essential to direct careful management but mostly based on clinical signs and symptoms, including the epidemiologic history, such as live-in/ travel-to an endemic area is a problem. To avoid complications typically seen between the fourth and sixth days of the disease, treatment administration has to be carried out earlier in the course of the disease. A study in Vietnamese adults with a history of 72 hours or less of dengue infection with treatment using chloroquine found no shortened viremia or NS1 antigenemia and no clinical benefit in dengue patients. ${ }^{22}$ A study of oral short-course prednisolone in Vietnamese patients with early dengue infection (clinically-diagnosed dengue and fever) for less than 72 hours found no correlation with any major adverse clinical or virological effects, nor with a decrease in the complications of dengue diseases. ${ }^{23}$ Both studies indicate that any treatment will likely start very early 
Table 1. Integrated management strategies for early DENV infection consist of four-dimensional aspects

\begin{tabular}{|c|c|c|c|c|}
\hline Strategy & Agents & Dosage & Benefit & Side effects \\
\hline $\begin{array}{l}\text { Rehydration } \\
\text { with ORT }\end{array}$ & ORS & $\begin{array}{l}\text { Ad libitum or as much } \\
\text { as one desires }\end{array}$ & $\begin{array}{l}\text { Correction and prevention } \\
\text { dehydration }\end{array}$ & $\begin{array}{l}\text { Vomiting and } \\
\text { diarrhea if too much } \\
\text { or rapid drinking }\end{array}$ \\
\hline \multirow{2}{*}{$\begin{array}{l}\text { Reduction of the } \\
\text { proinflammatory } \\
\text { cytokines, including } \\
\text { TNF- } \alpha \text { and IL- } 6\end{array}$} & Pentoxifylline & $\begin{array}{l}10 \mathrm{mg} / \mathrm{kg} \text { twice daily } \\
\text { (max. per dose } 400 \mathrm{mg} \\
\text { twice daily) for } 3 \text { days }\end{array}$ & $\begin{array}{l}\text { Inhibition of TNF- } \alpha \text {, IL-1, and } \\
\text { IL-6; Reduction in fever intensity; } \\
\text { Prevention of thrombocytopenia }\end{array}$ & $\begin{array}{l}\text { Upset stomach, } \\
\text { nausea, and vomiting }\end{array}$ \\
\hline & Doxycycline & $\begin{array}{l}2.5 \mathrm{mg} / \mathrm{kg} \text { once daily } \\
\text { (max. per dose } 100 \mathrm{mg} \\
\text { once daily) for } 3 \text { days }\end{array}$ & $\begin{array}{l}\text { Reduction in TNF- } \alpha \text { and } \\
\text { IL- } 6 \text { level; Prevention of } \\
\text { thrombocytopenia and } \\
\text { progression to severe dengue }\end{array}$ & $\begin{array}{l}\text { Gastritis, nausea, } \\
\text { and vomiting }\end{array}$ \\
\hline \multirow{2}{*}{$\begin{array}{l}\text { Inhibition of DENV } \\
\text { replication, viral } \\
\text { load reduction, } \\
\text { NS1Ag clearance }\end{array}$} & Doxycycline & $\begin{array}{l}2.5 \mathrm{mg} / \mathrm{kg} \text { once daily } \\
\text { (max. per dose } 100 \mathrm{mg} \\
\text { once daily) for } 3 \text { days }\end{array}$ & $\begin{array}{l}\text { Inhibition of DENV replication; } \\
\text { Reduction of the illness duration }\end{array}$ & $\begin{array}{l}\text { Gastritis, nausea, } \\
\text { and vomiting }\end{array}$ \\
\hline & Ivermectin & $\begin{array}{l}400-600 \mu \mathrm{g} / \mathrm{kg} / \text { dose } \\
\text { once daily for } 3 \text { days }{ }^{18}\end{array}$ & $\begin{array}{l}\text { Inhibition of nuclear transport } \\
\text { proteins (importin } \alpha / \beta 1 \text { ) for } \\
\text { viral replication; Decrease } \\
\text { in NS1 clearance time }\end{array}$ & None \\
\hline \multirow[t]{2}{*}{$\begin{array}{l}\text { Restoration of } \\
\text { immune function }\end{array}$} & Vitamin D & $\begin{array}{l}2,000 \mathrm{IU} / \mathrm{kg} / \text { day for } 3 \text { days } \\
\text { (max. per dose } 50,000 \\
\text { IU once daily for } 3 \text { days) } \\
\text { (pharmacological doses) }\end{array}$ & $\begin{array}{l}\text { Reduction in the production } \\
\text { of TNF- } \alpha \text { and NF-kB as well as } \\
\text { the inflammatory cytokines } \\
\text { IL- } 6, \text { IL- } 8 \text {, IL-12, and IL-17; } \\
\text { Control of dengue progression }\end{array}$ & None \\
\hline & $\begin{array}{l}\text { Zinc (zinc chelated } \\
\text { for better } \\
\text { absorption) }\end{array}$ & $\begin{array}{l}\text { Children >6 months of age: } 20 \\
\text { mg/day; Adults: } 50-100 \text { mg/ } \\
\text { day (pharmacological doses) }\end{array}$ & $\begin{array}{l}\text { Recovery of impaired } \\
\text { immune cell function; Early } \\
\text { recovery of the disease }\end{array}$ & None \\
\hline
\end{tabular}

during the course of the disease for favorable results and clinical benefit. Because plasma viremia tends to peak within 24-48 hours of fever onset and the occurrence of plasma leakage could begin as early as the third day of the disease from the ultrasonographic study, ${ }^{16}$ any intervention that can cause a positive clinical effect should be initiated within 24-48 hours after fever onset and also the first or second time to seek medical treatment in most patients. Successful therapies tend to need early treatment for providing physiologically beneficial responses. In summary, screening, diagnosis, and initiation of treatment with integrated management strategies would all occur within 24-48 hours of the onset of fever, which is considered a golden period of treatment.

\section{Importance of screening and confirmatory tools}

The traditional tourniquet test was utilized as a dengue diagnostic sign, according to the WHO 2009 guidelines for dengue. Nonetheless, a meta-analysis of 16 trials showed that the diagnostic efficiency was low, with a sensitivity and specificity of $58 \%$ and $71 \%$ respectively; although, there was a high level of bias in the publications. The low biological relation between dengue infection and capillary fragility could be the cause of the low diagnostic efficacy of the tourniquet test. ${ }^{24}$

Laboratory tests require a complete blood count (CBC) to differentiate and confirm the diagnosis. The $\mathrm{CBC}$ in patients with dengue infection changes with fever days, especially on days 3 to 8 , beginning with progressive leukopenia accompanied by thrombocytopenia and hemoconcentration caused by vascular leakage. ${ }^{25}$ Thrombocytopenia is not an initial predictor for DHF because the platelet counts in the early febrile stage of DF and DHF do not differ significantly. The platelet count is a tool to monitor the progress of the disease more than the early indicator for severe disease. NS1 Ag is a highly-conserved glycoprotein produced from infected cells and can be examined to detect dengue infection. It exists in the acute viremic phase of infection as opposed to immunoglobulin $\mathrm{M}(\mathrm{IgM})$ and immunoglobulin $\mathrm{G}(\mathrm{IgG})$.

Because the NS1Ag can be early detected, specific to dengue, and a large amount in the sera, it is suitable for early diagnosis and helps to inform clinical management. These features make NS1Ag the best choice for rapid diagnostic and confirmatory testing. The NS1Ag diagnostic kits vary extensively in sensitivity and specificity. ${ }^{24}$ The diagnostic sensitivity of the NS1Ag test in the febrile stage will exceed $90 \%$ for primary infections. In the febrile stage, the sensitivity of the NS1 Ag test is less than in the secondary infection (60-80\%), indicating the anamnestic immune response caused by previous DENV infection. ${ }^{26}$ In practice, the doctors cannot screen every patient showing acute febrile illness with the dengue NS1Ag tests, especially if the CBC did not suggest the existence of leukopenia, because it is wasteful and not worthwhile.

We have recently demonstrated invisible facial flushing in two cases of dengue infection and influenza by using PC software and a smartphone application: decorrelation stretching and K-means clustering. ${ }^{27}$ This innovative method is capable of showing invisible face flushing in dengue infection by using image enhancement with decorrelation stretching combined image segmentation with $\mathrm{K}$-means clustering. From the National Aeronautics and Space Administration's technology: decorrelation stretch to android application (Rock Art Enhancer App) was applied to the novel medical innovation for screening tools. With this method, we could use the enhanced single face photo as a new screening method for the diagnosis of dengue infection and influenza by smartphone appli- 
cation. ${ }^{28}$ According to our experience, the sensitivity of the threestep technique for all screening tests in dengue is up to $97.8 \%$. Thus, this innovative method will be a new screening tool and assist all medical providers to get an effective screening test in conjunction with the conventional method for the early diagnosis and detection of dengue infection.

\section{Early management proposal of dengue infection within 24-48 hours of fever onset}

\section{Rehydration with oral rehydration solution (ORS)}

Dengue patients tend to be vulnerable to dehydration due to high fever, nausea, and vomiting, altogether. ORT should be used for patients with moderate dehydration due to high fever and vomiting. In a study from a healthcare center in Nicaragua, the amount of fluid intake in the 24-hour period before coming to see a doctor with a high fluid intake at home could decrease the necessity for hospitalization of DF patients. ${ }^{29}$ This study indicated that maintaining hydration may result in a decrease in hospitalizations of patients with DF/DF with hemorrhagic manifestations. The "persistent vomiting" in the WHO 2009 is a warning sign for dengue case classification, in which one study suggested that the definition of permanent vomiting should be two or more vomits per day. ${ }^{30}$

Therefore, after one time of vomiting, the patient should be recommended to avoid eating food and replaced their diet with ORS to prevent further vomiting and developing even more dehydration. As for the WHO dengue guidelines (2012), dengue patients are advised to use multiple oral solutions, instead of using plain water for fluid treatment. ${ }^{6}$ One small study demonstrated the tendency that oral isotonic solution (OIS) to be acceptable and efficient, as opposed to plain water for dengue patients. ${ }^{31}$ The amount of oral fluids received is ad libitum or as much as one desires.

\section{Pentoxifylline}

Pentoxifylline is a nonspecific phosphodiesterase inhibitor, a wellestablished hemorheological factor, and functions for suppressing TNF- $\alpha$ production in macrophages via the cyclic AMP pathway. ${ }^{32}$ Pentoxifylline can inhibit the dose-dependent production of TNF- $\alpha$ at both the messenger ribonucleic acid (mRNA) level and the bioactivity level. ${ }^{33}$ Pentoxifylline is a widely available oral anti-TNF agent with a history of safety for over 50 years, and many studies have confirmed that pentoxifylline is an important inhibitor against TNF in the treatment of alcoholic hepatitis, over the last decade. ${ }^{34}$ Pentoxifylline inhibits the synthesis of various proinflammatory cytokines (TNF- $\alpha$, IL-1, IL-6) and prevents T lymphocyte cells and neutrophils from activating, proliferating, adhering, polarizing, and chemotaxis. ${ }^{35}$ Pentoxifylline has an anti-inflammatory role in the development of proinflammatory cytokines, including TNF- $\alpha$, IL-1, and IL- $6 .{ }^{36}$ TNF- $\alpha$ as well as IL- 1 are the main endogenous pyrogens, so using the pentoxifylline in dengue-infected patients may help in the reduction of fever intensity and the defervescence period.

In a pilot study, pentoxifylline has been demonstrated to reduce the proinflammatory actions of TNF- $\alpha$, an important cytokine of DHF. TNF- $\alpha$ is a proinflammatory cytokine that has been linked with the vascular leakage phenomenon. The TNF- $\alpha$ inhibitor pentoxifylline elicited a substantial decrease in the average duration of intensive care unit (ICU) stay and decreased TNF- $\alpha$ levels. ${ }^{19}$ The key finding showed a significant decrease in TNF- $\alpha$ values at 24 hours after the administration of pentoxifylline, in comparison to the control group. The result was more obvious in the subgroup of patients with greater-than grade III DHF, a group that is more likely to benefit from treatment because of the significance of TNF- $\alpha$ in plasma leakage.

Studies of the TNF- $\alpha$ blocking agent pentoxifylline have shown satisfactory results in child populations for a variety of pathologies, such as Kawasaki disease. Because TNF- $\alpha$ plays a major role in the inflammatory process in Kawasaki disease, pentoxifylline has been evaluated as a complementary drug in standard therapy. ${ }^{37} \mathrm{~A}$ study recently demonstrated the pharmacokinetics of an oral pediatric suspension of pentoxifylline in children diagnosed with acute Kawasaki disease. ${ }^{38}$ This drug was well tolerated and did not cause any toxic effects. The studies in preterm infants with sepsis found fewer complications with reduced mortality in the pentoxifyllinetreated cases. ${ }^{39}$ Pentoxifylline is an oral drug, readily available at an affordable price; so, it is useful in treating many inflammatory diseases, especially in countries with limited resources. The most common side effects of pentoxifylline are gastrointestinal disturbances, such as the upset stomach, nausea, and vomiting, which can be lessened by taking it with meals. If the theoretical treatment benefits of pentoxifylline for DENV infection can be demonstrated clinically, it would be a cost-effective, readily available, and useful treatment strategy to attack unfavorable cytokine overproduction in this disease.

\section{Doxycycline}

Doxycycline is a semi-synthetic derivative of tetracycline, as well as the well-defined antibiotic effect at high dose (100 or $200 \mathrm{mg} /$ day), in vitro studies, both high-dose and low-dose doxycycline (20-40 mg/day) have anti-inflammatory effects, inhibiting action on metalloproteases and modulating effects of IL-6, IL-8, and TNF- $\alpha$ proinflammatory cytokines. ${ }^{40}$ However, better efficacy of low-dose doxycycline, rather than high-dose, in the prevention of induced proinflammatory cytokines (i.e. IL-6) in inflammatory diseases were shown in the study. Doxycycline is quickly absorbed and almost completely after oral administration. It is a widely available and cheap drug, with a good safety profile. Digestive system conditions (nausea, vomiting, diarrhea, stomach pain, gastritis) and skin disorders (rash, sensitivity to sunlight) are the most common side effects.

In the past, doxycycline was contraindicated during pregnancy, due to its possible harmful teratogenic consequences, including in children under the age of 8 due to the possibility of yellow teeth discoloration and hypoplasia of the dental enamel. To date, recommendations have changed about doxycycline but not for other tetracyclines, which should be used for short courses $(<21$ days $)$, irrespective of age. ${ }^{41}$ Therefore, doxycycline is safe for short-term use in children of all ages, without concerns of tooth discoloration or tooth enamel weakening. ${ }^{42}$

Doxycycline, in addition to its immunomodulatory effect, has demonstrated ability to inhibit DENV replication in vitro by reducing the function and entry of viral proteases into the host cell, with particularly high activity against DENV2 and DENV4 in comparison to DENV1 and DENV3.43,44 Oral administration of doxycycline in patients with DF resulted in significantly reduced cytokine levels of IL-6 and TNF- $\alpha .^{45}$ In DHF cases, doxycycline decreased proinflammatory cytokines, including IL- 6 and TNF- $\alpha$, and the mortality rate was $46 \%$ less in doxycycline groups $(11.2 \%)$ than in untreated groups $(20.9 \%){ }^{46}$ Furthermore, in decreasing such proinflammatory cytokines, doxycycline was more powerful than tetracycline ${ }^{47}$ Doxycycline was very efficient in control- 
ling cytokine and cytokine receptor/antagonist levels and may be clinically useful for managing DENV infection by attenuating the cytokine cascade. Consequently, the anti-inflammatory and immunomodulatory effects of doxycycline may help to diminish the intensity of clinical symptoms and prevent progression of DENV infection to severe dengue, such as severe DHF and DSS.

\section{Ivermectin}

Ivermectin was discovered in the late 1970s and was developed by Satoshi Ōmura and William C. Campbell, who earned the Nobel Prize in Physiology or Medicine in 2015. ${ }^{48}$ Ivermectin has been prescribed for a long time in the treatment of infectious diseases in mammals, having high lipid solubility, with various impacts on parasites, nematodes, and arthropods. In addition to having antiparasitic activity, ivermectin offers several other potential effects, including antimicrobial, antiviral, and anticancer, as well as immunomodulation in the host.

Ivermectin exerts antiviral functions by inhibiting host nuclear import receptors importin- $\alpha$ and importin- $\beta$ (importin $\alpha / \beta 1$ nuclear transport proteins). These receptors are essential for DENV NS5 migration to the nucleus for efficient replication. ${ }^{49}$ Ivermectin is efficient on several enveloped, positive-sense, single-stranded RNA viruses, as well as severe respiratory syndrome coronavirus-2 (SARS-CoV-2). Over the past 50 years, numerous studies, both in vitro and in vivo, found ivermectin to have antiviral activity on RNA viruses, including Zika, dengue, yellow fever, West Nile virus, Chikungunya and Avian influenza A, etc. ${ }^{50,51}$ In one study, ${ }^{50}$ addition of the drug before the first 14 hours of entry of the virus into the cell demonstrated a stronger antiviral effect against the yellow fever virus, and this effect reduced significantly after the onset of intracellular RNA synthesis. Therefore, ivermectin may be effective in the initial stages of infection and should be used for the prevention or treatment of viral infections in the early stages rather than in the late stages. In the study of nuclear localization of DENV1-4 NS5, the inhibitor ivermectin's defense worked against all 4 DENV serotypes, disclosing its inhibitory impact on the possible function of ivermectin as an antiviral agent in the treatment of DENV infection at the importation of $\alpha / \beta$-mediated nuclear import. ${ }^{52}$

A phase III study of a once-daily dosage of oral ivermectin $400 \mu \mathrm{g} / \mathrm{kg}$ for 3 days compared to placebo for treating dengue infected patients, aged 15 years or older in Thailand, found it to be safe. Important differences in the NS1 clearance time and the percentage of patients with NS1 negativity at discharge within the two treatment groups indicated virological effectiveness. Nevertheless, ivermectin did not demonstrate therapeutic effectiveness in this study, ${ }^{21}$ which may be from use in the late stages (duration of presence of fever $\leq 72$ hours) and inpatient cases after intracellular viral RNA synthesis and replication. A clinical trial of pharmacokinetics and pharmacodynamics of ivermectin in pediatric dengue patients is now ongoing. All pediatric dengue patients will be administered ivermectin at $400-600 \mu \mathrm{g} / \mathrm{kg}$ every 24 hours for a total of three times. This clinical trial will study the effects of ivermectin on viral load or viremia clearance and NS1 antigenemia clearance. ${ }^{53}$

Ivermectin was accepted for the treatment of scabies in adults and children, but usage is off-label in infants and children weighing $15 \mathrm{~kg}$ or under. A multicentric observational study in infants and children below $15 \mathrm{~kg}$ with scabies and treated with oral ivermectin revealed the safety and effectiveness of ivermectin for the management of infants and young children with that disease. ${ }^{54}$ Of the 170 infants and children weighing less than $15 \mathrm{~kg}$, just seven individuals who were treated for oral ivermectin scabies identified minor adverse effects and no severe ones. Therefore, oral ivermectin has a very good safety profile with minimal side effects.

\section{Vitamin D supplementation}

Vitamin D is an immunomodulating hormone with anti-inflammatory and antimicrobial activity, and vitamin D is considered to play an important role in the immune system. ${ }^{18}$ The vitamin D receptor has been discovered in many organs and tissues, including cells in the brain, breast, lung, colon, bone marrow, and immune cells, both macrophages and white blood cells.

Vitamin D could diminish the production of $\mathrm{T}$ helper type 1 (Th1) cells and reduce the pro-inflammatory cytokines by inhibiting inflammatory progression and decreasing the production of inflammatory cytokines, such as IL-6, IL-8, IL-12, and IL-17. ${ }^{55}$ Vitamin $\mathrm{D}$ also reduces the production of TNF- $\alpha$ and nuclear factor-kappa $\mathrm{B}$ (NF-kB). Also, 1,25(OH)2D, a biologically active form of vitamin $\mathrm{D}$, specifically inhibits interferon-gamma (IFN- $\gamma$ ) and IL-2. The importance of vitamin D in the skeletal system is well established. New data suggests that it plays an essential role in immune system regulation, such as the immune response to viral infections. ${ }^{56}$

Vitamin D3 has been identified as an essential immune response modulator for several pathogenic agents. Interestingly, there is increasing evidence of vitamin D relation to decreased DENV infection and early recovery of the disease. Many studies have shown the association of vitamin D with significantly reduced DENV infection and early recovery of the disease. Puerta-Guardo et al. ${ }^{57}$ studied the impact of vitamin D3 treatment on two types of dengue-infected human cell lines (hepatic Huh-7 and monocytic U937). That study showed that response to 1,25-dihydroxy vitamin D3 decreased the amount of infected cells significantly, especially in monocytic cells, and reduced the generation of proinflammatory cytokines. Vitamin D3 significantly diminished the levels of proinflammatory cytokines (TNF- $\alpha$, IL-6, IL-1 $\beta$, etc.) released by the infected U937 cells. Such findings indicate vitamin D3 may be a beneficial antiviral agent. There is also a report on the relationship between the vitamin D receptor gene polymorphism and the possibility of symptomatic dengue requiring hospitalization. ${ }^{58}$ Arboleda Alzate et al..$^{59}$ found that monocyte-derived macrophages (referred to here as MDMs) differentiated in the presence of vitamin $\mathrm{D}$, as well as expressing lower mannose receptor levels, being less vulnerable to DENV infection and generating low levels of proinflammatory cytokines in comparison to MDMs differentiated in the absence of vitamin D. Therefore, differentiated human macrophages in the presence of vitamin D3 inhibit DENV infection and the innate responses by reducing mannose receptor function. The molecular mechanism study shows that vitamin D ameliorates the development of severe disease with DENV infection, and highlights its importance as a candidate for the prevention or treatment of the disease.

Vitamin D supplementation at high doses is linked with lower susceptibility to DENV infection from MDMs and proinflammatory cytokine production. ${ }^{60}$ The results of an exploratory study showed that vitamin D can also suppress DENV infection and improve the down-regulation of proinflammatory cytokine expression, while improving anti-inflammatory cytokine production. In this study, vitamin D supplementation at 4,000 IU/day was a sufficient dose to control dengue progression and dengue replication. MartínezMoreno et al. ${ }^{61}$ investigated the outcome of large doses of DENV replication vitamin D treatment, toll-like receptor expression, and dendritic cell cytokine profiles. In summary, the administration of vitamin $\mathrm{D}$ at 4,000 IU/day in 20 healthy donors challenged with 
DENV-2 reduced the DENV-2 infection, and this finding supports the important role of vitamin $\mathrm{D}$ in enhancing the innate immune response to DENV. Such findings indicate that increased supplementation of vitamin D may be a way of reducing the proinflammatory activity observed in severe dengue patients and could be used as a treatment strategy to control the progression of dengue infection.

Zaman et al. ${ }^{62}$ studied the efficacy of vitamin D in the prevention of DHF and DSS. Patients diagnosed with DF participated in this comparative analysis. The patients were split into two groups, each having 62 patients. Group A received a single dose of 200,000 IU vitamin D and group B received none of it. In that study, the group receiving vitamin D had less progressive DF to DHF. In conclusion, vitamin D reduced the risk of DHF and may be useful in managing DF. Therefore, all studies show the relationship between vitamin D and the clinical results of DENV infection, including reduced levels of proinflammatory cytokines.

\section{Zinc supplementation}

Zinc is important for the growth and development of all human beings. Previous studies have shown that zinc exhibits a range of direct and indirect antibiotic activities that are realized through different pathways. The administration of zinc supplements can increase antiviral protection, both innate and humoral, to maintain deficient immune cell activity or to boost normal immune cell activity, especially in patients who are immunocompromised or elderly. Zinc is necessary for a healthy immune system function. ${ }^{63}$

Zinc has been implicated in numerous biological processes and has different antiviral effects, both direct and indirect. The first line of defense is innate immunity, which means natural defense to infection. Innate immunity activities are impaired by changing zinc levels. Zinc deficiency is related to increased susceptibility to bacterial, virus, and fungal infections. The proper administration of zinc supplements in adequate therapeutic doses can recover impaired immune cell function or enhance regular immune cell function. ${ }^{64}$ It is estimated that zinc deficiency occurs in approximately one-third of the population in the world, estimated from $4 \%$ to $73 \%$ across sub-regions. A mild to moderate deficiency of zinc is widespread worldwide, while severe zinc deficiency is uncommon. ${ }^{65}$ Zinc deficiency in low-income countries is very common, which has a huge impact on children's health, especially as it impairs the immune system and causes infection.

In a study in Thailand, zinc deficiency in children with DENV infection revealed that zinc deficiency with dengue disease is widespread and patients with low zinc levels have a slightly longer period of fever and length of hospitalization than children with basal normal levels. ${ }^{66}$ A study of zinc supplementation as an adjunctive treatment for DENV infection in Thai children revealed that $46 \%$ of children admitted with DF/DHF had a zinc deficiency ${ }^{67}$ These children had a longer hospitalization than those with regular zinc levels, regardless of zinc supplementation. Although the time to defervescence does not benefit from zinc supplementation, children with basal normal zinc levels and with zinc supplementation demonstrated a shorter duration of fever. Ultimately, that study showed that zinc supplementation at admission to hospital for dengue disease might lead to reducing staying in the hospital.

\section{Early diagnosis and early management in dengue infection: a possible applied strategy to coronavirus disease (COVID-19)}

The global situation of COVID-19, which is considered to be the official pandemic, is likely to continue to rise. It has involved at least 213 countries, and more than 50,000,000 people worldwide have been infected, including deaths of more than 1,200,000 people. ${ }^{68}$ Early detection of COVID-19 is important for the treatment and prevention of infectious diseases. There is currently no standard treatment or available vaccine for COVID-19; therefore, we propose integrated management strategies for the early outpatient management of COVID-19 infection.

Both the clinical characteristics of COVID-19 and immunopathogenesis are comparable to dengue infection, particularly the hypercytokinemia (a "cytokine storm"), ${ }^{69-71}$ characterized by excessive secretion of cytokines in great amounts and elevated levels of proinflammatory cytokines, similar to emerging pathogenic viruses, like Ebola and the Middle Eastern coronavirus respiratory syndrome (known as MERS-CoV). ${ }^{72-75}$ From our experience in early management of dengue infection, we have successfully treated early dengue infection with early recovery results and without the progression to severe disease. Therefore, integrated management strategies for COVID-19 infection can also be applied in the same manner as with dengue infection. Therapeutic approaches based on these strategies include the following:

1. Reduction of the proinflammatory cytokines, including TNF- $\alpha$ and IL- $6^{73-75}$ with oral cytokine inhibitors (i.e. pentoxifylline $\mathrm{e}^{76-80}$ and doxycycline $\mathrm{81}^{86}$ ).

2. Inhibition of COVID-19 replication and viral load reduction with doxycycline ${ }^{86-91}$ and ivermectin. ${ }^{89-102}$

3. Restoring the immune function with vitamin $\mathrm{D}^{103-111}$ and zinc supplementation. ${ }^{112-115}$

From the citations of proposed COVID-19 treatments up to the present, there are more than 40 literature reviews and research studies that attempt to reason and explain the possibility of a treatment for COVID-19 with the same agents as in our proposed management of early dengue infection.

Also, there are more than 10 countries ${ }^{116}$ which have protocol and clinical trial studies for treatment of COVID-19 showing good results and low mortality rates by one or more agents, among five agents, as described:

1. Argentina: Ivermectin for treatment and prevention

2. Australia: Triple therapy with ivermectin plus doxycycline and zinc

3. Bangladesh: Double therapy with ivermectin plus doxycycline

4. Bolivia: Ivermectin for treatment

5. Brazil: Ivermectin plus vitamin D

6. Dominican Republic: Ivermectin for treatment

7. El Salvador: Ivermectin plus zinc and vitamin D in protocol treatment

8. Ethiopia: Ivermectin to prevent river blindness and COVID-19

9. Guatemala: Ivermectin plus zinc and vitamin D in protocol treatment

10. India: Quadruple therapy with ivermectin plus doxycycline, zinc, and vitamin D

11. Peru: Ivermectin in the treatment protocol

12. USA: South Florida: Ivermectin for treatment (ICON study); California: Clinical trial of combination therapy to treat COVID-19 infection (Ivermectin + doxycycline + zinc + vi$\operatorname{tamin} \mathrm{D}+\operatorname{vitamin} \mathrm{C})$

\section{Future directions}

The incidence of dengue has grown rapidly over the last decade around the world. About half of the world's population is now at 
risk. Each year, approximately 100-400 million infections occur. ${ }^{117}$ Earlier this year, WHO stated that dengue is a potential threat among the top 10 diseases for the year 2019, and the current outbreak in many countries confirms this observation. ${ }^{118}$ Overall average costs were I\$ (international dollars) 514 and I\$ 1,394 for outpatient and hospital cases respectively. The aggregate annual economic cost of dengue for the eight study countries, with an annual average of 574,000 patients documented, is no less than I\$ 587 million. ${ }^{119}$

Nowadays, treatment strategies for dealing with DENV infections as well as DF, DHF are only supportive. There is no particular treatment for dengue/severe dengue. Early diagnosis and early management proposal in dengue infection with integrated strategies within 24-48 hours after fever onset have a low risk and a significant potential yield; therefore, they require scientific validation. Four-dimensional aspects are proposed for addressing the early dengue infection to early recovery without complications and to prevent the disease progression to dengue fever and severe dengue, such as DHF, DSS, and EDS.

Meena et al. ${ }^{13}$ found that the increase in plasma TNF- $\alpha$ is linked to reduced platelet levels in clinical dengue infection; therefore, TNF- $\alpha$ is likely to play a role in enhancing DENV pathogenesis by contributing to the dengue-associated thrombocytopenia. Two oral cytokine inhibitors (i.e. pentoxifylline and doxycycline) can modulate proinflammatory levels of cytokines, including TNF- $\alpha$, IL-1, and IL-6, in the DENV-induced cytokine cascade to reduce and prevent DENV-induced thrombocytopenia in DF and DHF.

Doxycycline and ivermectin will inhibit DENV replication, resulting in reduced plasma virus load and NS1 antigenemia. Supplementation of vitamin D and zinc will be of beneficial effect to those with a deficiency, and vitamin D can cause a downregulation of proinflammatory cytokine function, while zinc can influence the immune response by restoring impaired immune cell activity or enhance the normal function of the immune cells. Both might help reduce the risk of disease progression associated with severe dengue and the severity of clinical symptoms.

From our 5-year (2015-2019) experience in early management of dengue infection, there are 23 dengue-infected patients, representing 14 males and 9 females. The mean age was 10 yearsold (range: 3-56 years) and the median age was 8 years-old. All patients were diagnosed and initiated on treatment within 24-48 hours after fever onset, following the integrated management strategies as outpatient cases. The earliest time of diagnosis and management was 2 hours after fever onset. All patients were successfully recovered within 4-5 days, without admission to the hospital. Most patients had a reduction in fever intensity within 24 hours and almost had fever clearance time within 48 hours after treatment. There was no hemoconcentration in any case, in which the changes of hematocrit were between $-5 \%$ to $+8 \%$. The platelet counts on the last day of treatment showed the lowest value as $177,000 \mathrm{cell} / \mathrm{mm}^{3}$ and the highest value as 364,000 cell/ $\mathrm{mm}^{3}$. All cases had recovered to the convalescent phase without any complications.

We expect benefits to result from early diagnosis and the early management proposal in dengue infection to achieve better both clinical and laboratory outcomes as follows:

1. Reduction in the defervescence period or fever clearance time and intensity of fever;

2. Reduction in the duration of illness to recovery or convalescent phase;

3. Lower incidence of DHF/DSS, decrease in the occurrence of shock or other known complications;

4. No plasma leakage or no critical phase; that is, the clinical symptoms will be better at the time of defervescence;

5. Reduction in the maximum percent hemoconcentration;

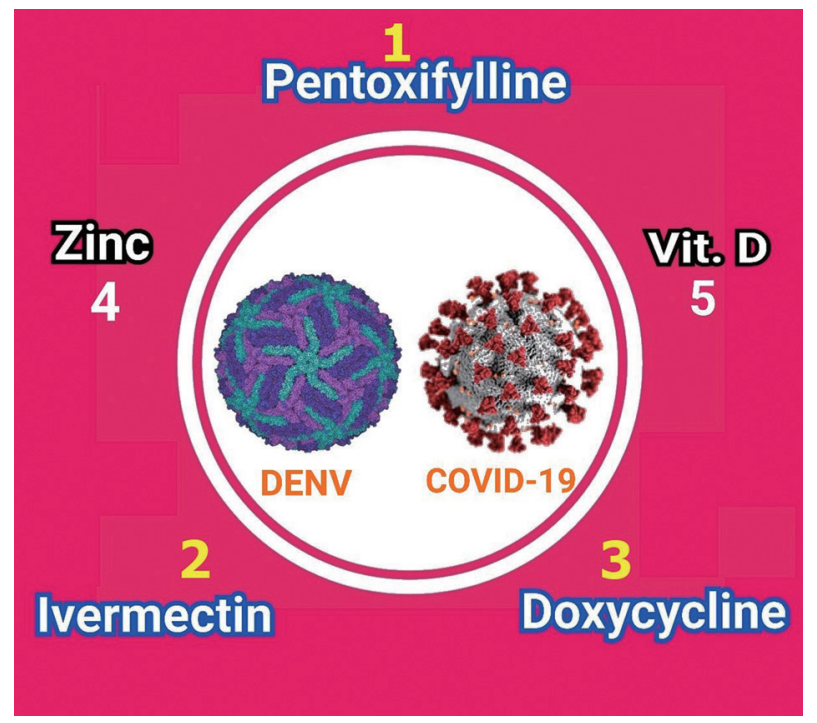

Fig. 1. A proposed protocol of five agents for early management in dengue infection and possibly COVID-19. Vit. D, vitamin D.

6. Minimal change of the minimum platelet count during the time of defervescence, so that dengue infection will eventually become UF or only viral infection, rather than DF and others;

7. Reduction in hospitalization and cost of treatment.

\section{Conclusions}

We believe that these hypotheses must be useful and possible for dengue infection, including other virus-induced hypercytokinemia, such as COVID-19 and Ebola. As such, with the protocol of the five agents, i.e., pentoxifylline, ivermectin, doxycycline, zinc, and vitamin D (Fig. 1), we can treat in the early stage of both dengue infection as well as COVID-19 simultaneously. It will be useful and greatly significant, especially in the case of COVID-19, in dengue-endemic countries which have co-infection cases. Finally, a randomized, controlled trial should be designed to assess the agents' ability to induce early recovery, prevent disease progression to severe dengue, and reduce the currently higher incidence of severe dengue-related morbidity and mortality.

\section{Acknowledgments}

We would like to thank Mrs. Sumalee Arpornsuwan for guidance and support in the publication of this study.

\section{Funding}

This research received no specific grant from any funding agency in the public, commercial, or not-for-profit sectors.

\section{Conflict of interest}

The authors declare that there is no conflict of interest. 
Author contributions

Conceptualization (MA, MA), methodology (MA, MA), validation (MA, MA), formal analysis (MA, MA), investigation (MA, MA), data curation (MA, MA), writing of the original draft (Manote A.), writing, review and editing of the manuscript (Matinun A.). All authors have made an intellectual contribution to the manuscript and approved the submission.

\section{References}

[1] Brady OJ, Gething PW, Bhatt S, Messina JP, Brownstein JS, Hoen AG, et al. Refining the global spatial limits of dengue virus transmission by evidence-based consensus. PLoS Negl Trop Dis 2012;6(8):e1760. doi:10.1371/journal.pntd.0001760.

[2] Bhatt S, Gething PW, Brady OJ, Messina JP, Farlow AW, Moyes $\mathrm{CL}$, et al. The global distribution and burden of dengue. Nature 2013;496(7446):504-507. doi:10.1038/nature12060.

[3] WHO. Dengue hemorrhagic fever: diagnosis, treatment, prevention, and control. 2nd ed. Geneva: World Health Organization; 1997. Available from: https://www.who.int/csr/resources/publications/dengue/ Denguepublication/en/. Accessed November 03, 2020.

[4] Sirivichayakul C, Limkittikul K, Chanthavanich P, Jiwariyavej V, Chokejindachai W, Pengsaa K, et al. Dengue infection in children in Ratchaburi, Thailand: a cohort study. II. Clinical manifestations. PLoS Negl Trop Dis 2012;6(2):e1520. doi:10.1371/journal.pntd.0001520.

[5] Rajapakse S, Rodrigo C, Rajapakse A. Treatment of dengue fever. Infect Drug Resist 2012;5:103-112. doi:10.2147/IDR.S22613.

[6] WHO, Special Programme for Research and Training in Tropical Diseases. Handbook for clinical management of dengue. Available from: https://www.who.int/denguecontrol/9789241504713/en/. Accessed November 03, 2020.

[7] Chunhakan S, Butthep P, Yoksan S, Tangnararatchakit K, Chuansumrit A. Vascular Leakage in Dengue Hemorrhagic Fever Is Associated with Dengue Infected Monocytes, Monocyte Activation/Exhaustion, and Cytokines Production. Int J Vasc Med 2015;2015:917143. doi:10.1155/2015/917143.

[8] Srikiatkhachorn A, Green S. Markers of Dengue Disease Severity. Curr Top Microbiol Immunol 2010;338:67-82. doi:10.1007/978-3-64202215-9_6.

[9] Halstead S. Recent advances in understanding dengue [version 1; peer review: 2 approved]. F1000 Faculty Rev 2019;8:1279. doi:10.12688/ f1000research.19197.1.

[10] Libraty DH, Endy TP, Houng HS, Green S, Kalayanarooj S, Suntayakorn $S$, et al. Differing influences of virus burden and immune activation on disease severity in secondary dengue-3 virus infections. J Infect Dis 2002:185(9):1213-1221. doi:10.1086/340365.

[11] Vaughn DW, Green S, Kalayanarooj S, Innis BL, Nimmannitya S, Suntayakorn $\mathrm{S}$, et al. Dengue viremia titer, antibody response pattern, and virus serotype correlate with disease severity. J Infect Dis 2000;181(1):2-9. doi:10.1086/315215

[12] Masood KI, Jamil B, Rahim M, Islam M, Farhan M, Hasan Z. Role of TNF $\alpha$, IL- 6 and CXCL10 in Dengue disease severity. Iran J Microbiol 2018;10(3):202-207.

[13] Meena AA, Murugesan A, Sopnajothi S, Yong YK, Ganesh PS, Vimali IJ, et al. Increase of Plasma TNF- $\alpha$ Is Associated with Decreased Levels of Blood Platelets in Clinical Dengue Infection. Viral Immunol 2020;33(1):54-60. doi:10.1089/vim.2019.0100.

[14] Kanwal F, Lu C, Qadri I, Sohail M, Farooqi ZR. Interleukin-6: A promising disease severity index for dengue virus infection. Asian Pac J Trop Dis 2017;7(5):266-269. doi:10.12980/apjtd.7.2017d6-461.

[15] Libraty DH, Young PR, Pickering D, Endy TP, Kalayanarooj S, Green S, et al. High circulating levels of the dengue virus nonstructural protein NS1 early in dengue illness correlate with the development of dengue hemorrhagic fever. J Infect Dis 2002;186(8):1165-8. doi:10.1086/ 343813.

[16] Srikiatkhachorn A, Krautrachue A, Ratanaprakarn W, Wongtapradit L, Nithipanya N, Kalayanarooj S, et al. Natural history of plasma leakage in dengue hemorrhagic fever: a serial ultrasonographic study. Pediatr Infect Dis J 2007;26(4):283-290; discussion 291-2. doi:10.1097/01. inf.0000258612.26743.10.

[17] Parthasarathy A. Textbook of Pediatric Infectious Diseases. Jaypee Brothers Medical Publishers; 2019.

[18] Aranow C. Vitamin D and the immune system. J Investig Med 2011;59(6):881-886. doi:10.2310/JIM.0b013e31821b8755.

[19] Salgado D, Zabaleta TE, Hatch S, Vega MR, Rodriguez J. Use of Pentoxifylline in Treatment of Children With Dengue Hemorrhagic Fever. Pediatr Infect Dis J 2012;31(7):771-773. doi:10.1097/ INF.0b013e3182575e6a.

[20] Castro JEZ, Vado-Solis I, Perez-Osorio C, Fredeking TM. Modulation of Cytokine and Cytokine Receptor/Antagonist by Treatment with Doxycycline and Tetracycline in Patients with Dengue Fever. Clin Dev Immunol 2011;2011:370872. doi:10.1155/2011/370872.

[21] Yamasmith E, Saleh-arong FA, Avirutnan P, Angkasekwinai N, Mairiang $\mathrm{D}$, Wongsawat $\mathrm{E}$, et al. Efficacy and safety of ivermectin against dengue infection: a phase III, randomized, double-blind, placebo-controlled trial. The 34th Annual Meeting the Royal College of Physicians of Thailand 'Internal Medicine and One Health' 2018. Available from: http://www.rcpt.org/abstractdb/media/abstract/CON2018/Best\%20 Resident27/BRA_77_Eakkawit.pdf. Accessed November 03, 2020.

[22] Tricou V, Minh NN, Van TP, Lee SJ, Farrar J, Wills B, et al. A randomized controlled trial of chloroquine for the treatment of dengue in Vietnamese adults. PLoS Negl Trop Dis 2010;4(8):e785. doi:10.1371/ journal.pntd.0000785.

[23] Tam DT, Ngoc TV, Tien NT, Kieu NT, Thuy TT, Thanh LT, et al. Effects of short-course oral corticosteroid therapy in early dengue infection in Vietnamese patients: a randomized, placebo-controlled trial. Clin Infect Dis 2012;55(9):1216-1224. doi:10.1093/cid/cis655.

[24] Raafat N, Blacksell SD, Maude RJ. A review of dengue diagnostics and implications for surveillance and control. Trans R Soc Trop Med Hyg 2019;113(11):653-660. doi:10.1093/trstmh/trz068.

[25] Oliveira EC, Pontes ER, Cunha RV, Froes IB, Nascimento Dd. Hematological abnormalities in patients with dengue (in Portuguese). Rev Soc Bras Med Trop 2009;42(6):682-685. doi:10.1590/s003786822009000600014.

[26] Guzman MG, Jaenisch T, Gaczkowski R, Ty Hang VT, Sekaran SD, Kroeger A, et al. Multi-country evaluation of the sensitivity and specificity of two commercially-available NS1 ELISA assays for dengue diagnosis. PLoS Negl Trop Dis 2010;4(8):e811. doi:10.1371/journal. pntd.0000811.

[27] Arpornsuwan M, Arpornsuwan M. Invisible Facial Flushing in Two Cases of Dengue Infection and Influenza Detected by PC Program and Smartphone App: Decorrelation Stretching and K-Means Clustering. Case Rep Infect Dis 2020;2020:8790130. doi:10.1155/2020/8790130.

[28] Arpornsuwan M, Arpornsuwan M. Application of the Enhanced Single Face Photo as a Novel Screening Tool for Diagnosis of Dengue Infection and Influenza. International Journal of Advance Research, Ideas and Innovation Technology 2020;6(2):715-724.

[29] Harris E, Pérez L, Phares CR, Pérez Mde L, Idiaquez W, Rocha J, et al. Fluid intake and decreased risk for hospitalization for dengue fever, Nicaragua. Emerg Infect Dis 2003;9(8):1003-1006. doi:10.3201/ eid0908.020456.

[30] Vuong NL, Manh DH, Mai NT, Phuc LH, Luong VT, Quan VD, et al. Criteria of "persistent vomiting" in the WHO 2009 warning signs for dengue case classification. Trop Med Health 2016;44:14. doi:10.1186/ s41182-016-0014-9.

[31] Nainggolan L, Bardosono S, Ibrahim Ilyas El. The Tolerability and Efficacy of Oral Isotonic Solution versus Plain Water in Dengue Patients: A Randomized Clinical Trial. Indian J Community Med 2018;43(1):2933. doi:10.4103/ijcm.IJCM 377_16.

[32] Deree J, Martins JO, Melbostad H, Loomis WH, Coimbra R. Insights into the regulation of TNF-alpha production in human mononuclear cells: the effects of non-specific phosphodiesterase inhibition. Clinics (Sao Paulo) 2008;63(3):321-328. doi:10.1590/s1807-59322 008000300006

[33] Strieter RM, Remick DG, Ward PA, Spengler RN, Lynch JP 3rd, Larrick $\mathrm{J}$, et al. Cellular and molecular regulation of tumor necrosis factoralpha production by pentoxifylline. Biochem Biophys Res Commun 1988;155(3):1230-1236. doi:10.1016/s0006-291x(88)81271-3. 
[34] Thursz M, Richardson P, Allison M, Austin A, Bowers M, Day CP, et al. Prednisolone or pentoxifylline for alcoholic hepatitis. N Engl J Med 2015;372(17):1619-1628. doi:10.1056/NEJMoa1412278.

[35] Kreth S, Ledderose C, Luchting B, Weis F, Thiel M. Immunomodulatory properties of pentoxifylline are mediated via adenosinedependent pathways. Shock 2010;34(1):10-16. doi:10.1097/SHK Ob013e3181cdc3e2.

[36] Bienvenu J, Doche C, Gutowski MC, Lenoble M, Lepape A, Perdrix JP. Production of pro-inflammatory cytokines and cytokines involved in the $\mathrm{TH} 1 / \mathrm{TH} 2$ balance is modulated by pentoxifylline. J Cardiovasc Pharmacol 1995;25 Suppl 2:S80-S84. doi:10.1097/00005344$199500252-00017$.

[37] Newburger JW, Takahashi M, Gerber MA, Gewitz MH, Tani LY, Burns $\mathrm{JC}$, et al. Diagnosis, treatment, and long-term management of Kawasaki disease: a statement for health professionals from the Committee on Rheumatic Fever, Endocarditis and Kawasaki Disease, Council on Cardiovascular Disease in the Young, American Heart Association. Circulation 2004;110(17):2747-2771. doi:10.1161/01. CIR.0000145143.19711.78.

[38] Best BM, Burns JC, DeVincenzo J, Phelps SJ, Blumer JL, Wilson JT, et al. Pharmacokinetic and tolerability assessment of a pediatric oral formulation of pentoxifylline in kawasaki disease. Curr Ther Res Clin Exp 2003;64(2):96-115. doi:10.1016/S0011-393X(03)00018-3.

[39] Adel M, Awad HA, Abdel-Naim AB, Al-Azizi MM. Effects of pentoxifylline on coagulation profile and disseminated intravascular coagulation incidence in Egyptian septic neonates. J Clin Pharm Ther 2010;35(3):257-265. doi:10.1111/j.1365-2710.2009.01077.x.

[40] Di Caprio R, Lembo S, Di Costanzo L, Balato A, Monfrecola G. Antiinflammatory properties of low and high doxycycline doses: an in vitro study. Mediators Inflamm 2015;2015:329418. doi:10.1155/ 2015/329418.

[41] Stultz JS, Eiland LS. Doxycycline and Tooth Discoloration in Children: Changing of Recommendations Based on Evidence of Safety. Ann Pharmacother 2019;53(11):1162-1166. doi:10.1177/ 1060028019863796.

[42] Hemphill ME, Mollanazar NK, Hsu S. Doxycycline Is Safe for ShortTerm Use in Children of All Ages. Skinmed 2019;17(5):322.

[43] Rothan HA, Buckle MJ, Ammar YA, Mohammadjavad P, Shatrah O, Noorsaadah AR, et al. Study the antiviral activity of some derivatives of tetracycline and non-steroid anti inflammatory drugs towards dengue virus. Trop Biomed 2013;30(4):681-690.

[44] Rothan HA, Mohamed Z, Paydar M, Rahman NA, Yusof R. Inhibitory effect of doxycycline against dengue virus replication in vitro. Arch Virol 2014;159(4):711-718. doi:10.1007/s00705-013-1880-7.

[45] Castro JE, Vado-Solis I, Perez-Osorio C, Fredeking TM. Modulation of cytokine and cytokine receptor/antagonist by treatment with doxycycline and tetracycline in patients with dengue fever. Clin Dev Immunol 2011;2011:370872. doi:10.1155/2011/370872.

[46] Fredeking TM, Zavala-Castro JE, González-Martínez P, MoguelRodríguez W, Sanchez EC, Foster MJ, et al. Dengue Patients Treated with Doxycycline Showed Lower Mortality Associated to a Reduction in IL-6 and TNF Levels. Recent Pat Antiinfect Drug Discov 2015;10(1):51-58. doi:10.2174/1574891x10666150410153839.

[47] Kanodia N, Khare DC. A Randomized Controlled Study to Assess the Effect of Doxycycline on IL-6 and TNF Levels among the Patients of Dengue. J Med Sci Clin Res 2018;6(2):1079-1083. doi:10.18535/jm$\mathrm{scr} / \mathrm{v} 6 \mathrm{i} 2.168$.

[48] Crump A, Omura S. Ivermectin, "Wonder drug" from Japan: the human use perspective. Proc Jpn Acad Ser B Phys Biol Sci 2011;87(2):1328. doi:10.2183/pjab.87.13.

[49] Yang SNY, Atkinson SC, Wang C, Lee A, Bogoyevitch MA, Borg NA, et $a l$. The broad spectrum antiviral ivermectin targets the host nuclear transport importin $\alpha / \beta 1$ heterodimer. Antiviral Res 2020;177:104760. doi:10.1016/j.antiviral.2020.104760.

[50] Mastrangelo E, Pezzullo M, De Burghgraeve T, Kaptein S, Pastorino B, Dallmeier K, et al. Ivermectin is a potent inhibitor of flavivirus replication specifically targeting NS3 helicase activity: new prospects for an old drug. J Antimicrob Chemother 2012;67(8):1884-1894. doi:10.1093/ jac/dks147.

[51] Varghese FS, Kaukinen P, Gläsker S, Bespalov M, Hanski L, Wennerberg $\mathrm{K}$, et al. Discovery of berberine, abamectin and ivermectin as antivirals against chikungunya and other alphaviruses. Antiviral Res 2016;126:117-124. doi:10.1016/j.antiviral.2015.12.012.

[52] Tay MY, Fraser JE, Chan WK, Moreland NJ, Rathore AP, Wang C, et al. Nuclear localization of dengue virus (DENV) 1-4 non-structural protein 5; protection against all 4 DENV serotypes by the inhibitor Ivermectin. Antiviral Res 2013;99(3):301-306. doi:10.1016/j.antiviral.2013.06.002.

[53] Avirutnan P, Mairiang D. Pharmacokinetics and Pharmacodynamics of Ivermectin in Pediatric Dengue Patients (PKIDEN). ClinicalTrials.gov; 2018. Available from: https://clinicaltrials.gov/ct2/show/NCT0343 2442. Accessed November 03, 2020.

[54] Levy M, Martin L, Bursztejn AC, Chiaverini C, Miquel J, Mahé E, et al. Ivermectin safety in infants and children under $15 \mathrm{~kg}$ treated for scabies: a multicentric observational study. $\mathrm{Br} J$ Dermatol 2020;182(4):1003-1006. doi:10.1111/bjd.18369.

[55] Skrobot A, Demkow U, Wachowska M. Immunomodulatory Role of Vitamin D: A Review. Adv Exp Med Biol 2018;1108:13-23. doi:10.1007/5584_2018_246.

[56] Beard JA, Bearden A, Striker R. Vitamin D and the anti-viral state. J Clin Virol 2011;50(3):194-200. doi:10.1016/j.jcv.2010.12.006.

[57] Puerta-Guardo H, Medina F, De la Cruz Hernández SI, Rosales VH, Ludert JE, del Angel RM. The 1 $\alpha, 25$-dihydroxy-vitamin D3 reduces dengue virus infection in human myelomonocyte (U937) and hepatic (Huh-7) cell lines and cytokine production in the infected monocytes. Antiviral Res 2012;94(1):57-61. doi:10.1016/j.antiviral.2012.02.006.

[58] Alagarasu K, Honap T, Mulay AP, Bachal RV, Shah PS, Cecilia D. Association of vitamin $D$ receptor gene polymorphisms with clinical outcomes of dengue virus infection. Hum Immunol 2012;73(11):11941199. doi:10.1016/j.humimm.2012.08.007.

[59] Arboleda Alzate JF, Rodenhuis-Zybert IA, Hernández JC, Smit JM, Urcuqui-Inchima S. Human macrophages differentiated in the presence of vitamin D3 restrict dengue virus infection and innate responses by downregulating mannose receptor expression. PLoS Negl Trop Dis 2017;11(10):e0005904. doi:10.1371/journal.pntd.0005904.

[60] Giraldo DM, Cardona A, Urcuqui-Inchima S. High-dose of vitamin D supplement is associated with reduced susceptibility of monocytederived macrophages to dengue virus infection and pro-inflammatory cytokine production: An exploratory study. Clin Chim Acta 2018;478:140-151. doi:10.1016/j.cca.2017.12.044.

[61] Martínez-Moreno J, Hernandez JC, Urcuqui-Inchima S. Effect of high doses of vitamin D supplementation on dengue virus replication, Toll-like receptor expression, and cytokine profiles on dendritic cells. Mol Cell Biochem 2020;464(1-2):169-180. doi:10.1007/s11010-01903658-w.

[62] Zaman S. Effectiveness of Vitamin D in Prevention of Dengue Haemorrhagic Fever and Dengue Shock Syndrome. Journal of Rawalpindi Medical College 2017;21(3):205-207.

[63] Rink L, Gabriel P. Zinc and the immune system. Proc Nutr Soc 2000;59(4):541-552. doi:10.1017/s0029665100000781.

[64] Overbeck S, Rink L, Haase H. Modulating the immune response by oral zinc supplementation: a single approach for multiple diseases. Arch Immunol Ther Exp (Warsz) 2008;56(1):15-30. doi:10.1007/ s00005-008-0003-8.

[65] Sandstead HH. Zinc deficiency. A public health problem? Am J Dis Child 1991;145(8):853-859. doi:10.1001/archpedi.1991.021600800 29016.

[66] Rerksuppaphol L, Rerksuppaphol S. Zinc deficiency in children with Dengue viral infection. Pediatr Rep 2019;11(1):7386. doi:10.4081/ pr.2019.7386.

[67] Rerksuppaphol S, Rerksuppaphol L. A Randomized Controlled Trial of Zinc Supplementation as Adjuvant Therapy for Dengue Viral Infection in Thai Children. Int J Prev Med 2018;9:88. doi:10.4103/ijpvm. IJPVM_367_17.

[68] Dong $\bar{E}, \mathrm{Du} \mathrm{H}$, Gardner L. An interactive web-based dashboard to track COVID-19 in real-time. Lancet Infect Dis 2020;20(5):533-534. doi:10.1016/S1473-3099(20)30120-1.

[69] Tisoncik JR, Korth MJ, Simmons CP, Farrar J, Martin TR, Katze MG. Into the Eye of the Cytokine Storm. Microbiol Mol Biol Rev 2012;76(1):16-32. doi:10.1128/MMBR.05015-11.

[70] Ye Q, Wang B, MaoJ. The pathogenesis and treatment of the 'Cytokine Storm' in COVID-19.J Infect. 2020;80(6):607-613. doi:10.1016/j. 
jinf.2020.03.037.

[71] Harapan H, Ryan M, Yohan B, Abidin RS, Nainu F, Rakib A, et al. Covid-19 and dengue: Double punches for dengue-endemic countries in Asia. Rev Med Virol 2020:e2161. doi:10.1002/rmv.2161.

[72] Wong JP, Viswanathan S, Wang M, Sun L-Q, Clark GC, D’Elia RV. Current and future developments in the treatment of virus-induced hypercytokinemia. Future Med Chem 2017;9(2):169-178. doi:10.4155/ fmc-2016-0181.

[73] Abdin SM, Elgendy SM, Alyammahi SK, Alhamad DW, Omar HA. Tackling the cytokine storm in COVID-19, challenges, and hopes. Life Sci 2020;257:118054. doi:10.1016/j.lfs.2020.118054.

[74] Lee KY, Rhim JW, Kang JH. Immunopathogenesis of COVID-19 and early immunomodulators. Clin Exp Pediatr 2020;63(7):239-250. doi:10.3345/cep.2020.00759.

[75] Sun X, Wang T, Cai D, Hu Z, Chen J, Liao H, et al. Cytokine storm intervention in the early stages of COVID-19 pneumonia. Cytokine Growth Factor Rev 2020;53:38-42. doi:10.1016/j.cytogfr.2020.04.002.

[76] Seirafianpour F, Mozafarpoor S, Fattahi N, Sadeghzadeh-Bazargan A, Hanifiha M, Goodarzi A. Treatment of COVID-19 with pentoxifylline: Could it be a potential adjuvant therapy? Dermatol Ther 2020;33(4):e13733. doi:10.1111/dth.13733.

[77] Arnold J, Stafford N, Arnold A, Sangwaiya A, Manglam V. Pentoxifylline Is An Inexpensive And Widely Available Oral Anti-Tumour Necrosis Factor Agent For Prevention Or Treatment Of Cytokine Storm In COVID-19. The BMJ 2020. Available from: http://www.bmj.com/ content/369/bmj.m1573/rr-0. Accessed November 03, 2020.

[78] Assimakopoulos SF, Seintis F, Marangos M. Pentoxifylline, and complicated COVID-19: A pathophysiologically based treatment proposal. Med Hypotheses 2020;143:109926. doi:10.1016/j.mehy. 2020.109926.

[79] Hendry BM, Stafford N, Arnold AD, Sangwaiya A, Manglam V, Rosen $\mathrm{SD}$, et al. Hypothesis: Pentoxifylline is a potential cytokine modulator therapeutic in COVID-19 patients. Pharmacol Res Perspect 2020;8(4):e00631. doi:10.1002/prp2.631.

[80] Maldonado V, Loza-Mejía MA, Chávez-Alderete J. Repositioning of pentoxifylline as an immunomodulator and regulator of the reninangiotensin system in the treatment of COVID-19. Med Hypotheses 2020;144:109988. doi:10.1016/j.mehy.2020.109988.

[81] Sodhi M, Etminan M. Therapeutic Potential for Tetracyclines in the Treatment of COVID-19. Pharmacotherapy 2020;40(5):487-488. doi:10.1002/phar.2395.

[82] Conforti C, Giuffrida R, Zalaudek I, Di Meo N. Doxycycline, a Widely Used Antibiotic in Dermatology with a Possible Anti-Inflammatory Action against IL-6 in COVID-19 Outbreak. Dermatologic Therapy 2020. doi:10.1111/dth.13437.

[83] Szolnoky G. Further aspects of doxycycline therapy in COVID-19. Dermatologic Therapy 2020. doi:10.1111/dth.13810.

[84] Malek AE, Granwehr BP, Kontoyiannis DP. Doxycycline as a potential partner of COVID-19 therapies. IDCases 2020;21:e00864. doi:10.1016/j.idcr.2020.e00864.

[85] Francini E, Miano ST, Fiaschi Al, Francini G. Doxycycline or minocycline may be a viable treatment option against SARS-CoV-2. Med Hypotheses 2020;144:110054. doi:10.1016/j.mehy.2020.110054.

[86] Alam MM, Mahmud S, Rahman MM, Simpson J, Aggarwal S, Ahmed Z. Clinical Outcomes of Early Treatment With Doxycycline for 89 High-Risk COVID-19 Patients in Long-Term Care Facilities in New York. Cureus 2020;12(8):e9658. doi:10.7759/cureus.9658.

[87] Sachdeva C, Wadhwa A, Kumari A, Hussain F, Jha P, Kaushik NK. In silico Potential of Approved Antimalarial Drugs for Repurposing Against COVID-19. OMICS 2020;24(10):568-580. doi:10.1089/ omi.2020.0071.

[88] Siddiqui AJ, Jahan S, Ashraf SA, Alreshidi M, Ashraf MS, Patel M, et al. Current status and strategic possibilities on potential use of combinational drug therapy against COVID-19 caused by SARS-CoV-2. J Biomol Struct Dyn 2020. doi:10.1080/07391102.2020.1802345.

[89] Alam MT, Murshed R, Bhiuyan E, Saber S, Alam RF, Robin RC. A Case Series of 100 COVID-19 Positive Patients Treated with Combination of Ivermectin and Doxycycline. Journal of Bangladesh College of Physicians and Surgeons 2020;38:10-15. doi:10.3329/jbcps.v38i0.47512.

[90] Rahman MA, Iqbal SA, Islam MA, Niaz MK, Hussain T, Siddiquee TH. Comparison of Viral Clearance between Ivermectin with Doxy- cycline and Hydroxychloroquine with Azithromycin in COVID-19 Patients. Journal of Bangladesh College of Physicians and Surgeons 2020;38:5-9. doi:10.3329/jbcps.v38i0.47514.

[91] Maurya DK. A Combination of Ivermectin and Doxycycline Possibly Blocks the Viral Entry and Modulate the Innate Immune Response in COVID-19 Patients. ChemRxiv (Preprint) 2020. doi:10.26434/chemrxiv.12630539.v1.

[92] Caly L, Druce JD, Catton MG, Jans DA, Wagstaff KM. The FDA-approved drug ivermectin inhibits the replication of SARS-CoV-2 in vitro. Antiviral Res 2020;178:104787. doi:10.1016/j.antiviral.2020.104787.

[93] Chaccour C, Hammann F, Ramón-García S, Rabinovich NR. Ivermectin and COVID-19: Keeping Rigor in Times of Urgency. Am J Trop Med Hyg 2020;102(6):1156-1157. doi:10.4269/ajtmh.20-0271.

[94] Bray M, Rayner C, Noël F, Jans D, Wagstaff K. Ivermectin and COVID-19: A report in Antiviral Research, widespread interest, an FDA warning, two letters to the editor and the authors' responses. Antiviral Res 2020;178:104805. doi:10.1016/j.antiviral.2020.104805.

[95] Rizzo E. Ivermectin, antiviral properties, and COVID-19: a possible new mechanism of action. Naunyn Schmiedebergs Arch Pharmacol 2020;393(7):1153-1156. doi:10.1007/s00210-020-01902-5.

[96] Wamae CN. Mass Drug Administration and Worms Experience in Africa: Envisage Repurposing Ivermectin for SARS-COV-2. Am J Trop Med Hyg 2020;103(1):10-11. doi:10.4269/ajtmh.20-0295.

[97] Sharun K, Dhama K, Patel SK, Pathak M, Tiwari R, Singh BR, et al. Ivermectin, a new candidate therapeutic against SARS-CoV-2/COVID-19. Ann Clin Microbiol Antimicrob 2020;19(1):23. doi:10.1186/s12941020-00368-w.

[98] Heidary F, Gharebaghi R. Ivermectin: a systematic review from antiviral effects to COVID-19 complementary regimen. J Antibiot (Tokyo) 2020;73(9):593-602. doi:10.1038/s41429-020-0336-z.

[99] Gupta D, Sahoo AK, Singh A. Ivermectin: a potential candidate for the treatment of COVID-19. Braz J Infect Dis 2020;24(4):369-371. doi:10.1016/j.bjid.2020.06.002.

[100] de Castro CG Jr, Gregianin LJ, Burger JA. Continuous high-dose ivermectin appears to be safe in patients with acute myelogenous leukemia and could inform clinical repurposing for COVID-19 infection. Leuk Lymphoma 2020;61(10):2536-2537. doi:10.1080/10428194.20 20.1786559.

[101] Lehrer S, Rheinstein PH. Ivermectin Docks to the SARS-CoV-2 Spike Receptor-binding Domain Attached to ACE2. In Vivo 2020;34(5):30233026. doi:10.21873/invivo.12134.

[102] Rajter JC, Sherman MS, Fatteh N, Vogel F, Sacks J, Rajter JJ. Use of Ivermectin Is Associated With Lower Mortality in Hospitalized Patients With Coronavirus Disease 2019: The ICON Study. Chest 2020. doi:10.1016/j.chest.2020.10.009.

[103] Grant WB, Lahore H, McDonnell SL, Baggerly CA, French CB, Aliano $\mathrm{JL}$, et al. Evidence that Vitamin D Supplementation Could Reduce Risk of Influenza and COVID-19 Infections and Deaths. Nutrients 2020;12(4):988. doi:10.3390/nu12040988.

[104] Panarese A, Shahini E. Letter: Covid-19, and vitamin D. Aliment Pharmacol Ther. 2020;51(10):993-995. doi:10.1111/apt.15752.

[105] McCartney DM, Byrne DG. Optimization of Vitamin D Status for Enhanced Immuno-protection Against Covid-19. Ir Med J 2020;113(4):58.

[106] Maestri E, Formoso G, Da Cas R, Mammarella F, Guerrizio MA, Trotta F. Vitamin D and coronavirus: a new field of use? Recent Progress in Medicine 2020. doi:10.1701/3347.33188.

[107] Zemb P, Bergman P, Camargo CA Jr, Cavalier E, Cormier C, Courbebaisse $\mathrm{M}$, et al. Vitamin $\mathrm{D}$ deficiency and the COVID-19 pandemic. J Glob Antimicrob Resist 2020;22:133-134. doi:10.1016/j. jgar.2020.05.006.

[108] Aygun H. Vitamin D can prevent COVID-19 infection-induced multiple organ damage. Naunyn Schmiedebergs Arch Pharmacol 2020;393(7):1157-1160. doi:10.1007/s00210-020-01911-4.

[109] Laird E, Rhodes J, Kenny RA. Vitamin D and Inflammation: Potential Implications for Severity of Covid-19. Ir Med J 2020;113(5):81.

[110] Orrù B, Szekeres-Bartho J, Bizzarri M, Spiga AM, Unfer V. Inhibitory effects of Vitamin D on inflammation and IL-6 release. A further support for COVID-19 management? Eur Rev Med Pharmacol Sci 2020;24(15):8187-8193. doi:10.26355/eurrev_202008_22507.

[111] Ohaegbulam KC, Swalih M, Patel P, Smith MA, Perrin R. Vitamin D 
Supplementation in COVID-19 Patients: A Clinical Case Series. Am J Ther 2020;27(5):e485-e490. doi:10.1097/MJT.0000000000001222.

[112] Derwand R, Scholz M. Does zinc supplementation enhance the clinical efficacy of chloroquine/hydroxychloroquine to win today's battle against COVID-19? Med Hypotheses 2020;142:109815. doi:10.1016/j.mehy.2020.109815.

[113] Kumar A, Kubota $Y$, Chernov M, Kasuya H. Potential role of zinc supplementation in prophylaxis and treatment of COVID-19. Med Hypotheses 2020;144:109848. doi:10.1016/j.mehy.2020.109848.

[114] Wessels I, Rolles B, Rink L. The Potential Impact of Zinc Supplementation on COVID-19 Pathogenesis. Front Immunol 2020;11:1712. doi:10.3389/fimmu.2020.01712.

[115] Carlucci PM, Ahuja T, Petrilli C, Rajagopalan H, Jones S, Rahimian J. Zinc sulfate in combination with a zinc ionophore may improve outcomes in hospitalized COVID-19 patients. J Med Microbiol 2020;69(10):1228-1234. doi:10.1099/jmm.0.001250.

[116] The Complete Guide To Health.com. Ivermectin. Available from: https://www.thecompleteguidetohealth.com/ivermectin.html. Accessed November 03, 2020.

[117] WHO. Dengue and Severe Dengue. Available from: http://www. who.int/news-room/fact-sheets/detail/dengue-and-severe-dengue. Accessed November 03, 2020.

[118] WHO. Dengue and Severe Dengue. Available from: http://www. who.int/health-topics/dengue-and-severe-dengue. Accessed November 03, 2020.

[119] Suaya JA, Shepard DS, Siqueira JB, Martelli CT, Lum LC, Tan LH, et al. Cost of dengue cases in eight countries in the Americas and Asia: a prospective study. Am J Trop Med Hyg 2009;80(5):846-855. 